\title{
O estudo de geometria esférica na formação de professores de matemática: uma experiência baseada na utilização de materiais manipuláveis
}

\author{
The study of spherical geometry in Mathematics teacher education: an experience based on the use \\ of manipulable materials \\ El estudio de la geometría esférica en la formación de profesores de Matemáticas: una experiencia \\ basada en el uso de materiales manipulables
}

Recebido: 02/01/2021 | Revisado: 03/01/2021 | Aceito: 05/01/2021 | Publicado: 07/01/2021

Isabela Cristina Soares Gregor
ORCID: https://orcid.org/0000-0001-5780-2408
Secretaria Estadual de Educação de Minas Gerais, Brasil
E-mail: isabellagregor@hotmail.com
Josué Antunes de Macêdo
ORCID: https://orcid.org/0000-0001-7737-7509
Instituto Federal de Educação, Ciência e Tecnologia do Norte de Minas Gerais, Brasil
Universidade Estadual de Montes Claros, Brasil
E-mail: josueama@gmail.com
Edson Crisostomo
ORCID: https://orcid.org/0000-0001-7078-243X
Universidade Estadual de Montes Claros, Brasil
E-mail: edson.crisostomo@unimontes.br
Daniel Martins Nunes
ORCID: https://orcid.org/0000-0002-2711-0272
E-mail: daniel.nunes@ifnmg.edu.br

\section{Resumo}

O presente trabalho aborda os principais resultados de uma pesquisa, cujo foco foi apresentar conceitos de Geometria Esférica por meio de materiais manipuláveis e atividades orientadas para os acadêmicos do curso de Licenciatura em Matemática. Para dar suporte teórico e conceitual à pesquisa, foram considerados parâmetros da utilização de materiais manipuláveis no ensino de Matemática e sobre o ensino de Geometria no Brasil. A metodologia utilizada buscou conciliar ideias sobre a utilização de materiais manipuláveis para a organização de atividades orientadas que permitissem aos estudantes realizarem descobertas por si. As atividades foram aplicadas a estudantes do curso de Licenciatura em Matemática de um Instituto Federal. Os resultados da pesquisa foram analisados qualitativamente, usando parâmetros do modelo de Van Hiele para descrever o desempenho dos envolvidos. A contribuição dos resultados desta pesquisa está inserida no contexto do ensino de Geometria, especificamente na utilização de materiais manipuláveis no processo de ensino e aprendizagem.

Palavras-chave: Formação de professores; Geometrias não euclidianas; Geometria esférica; Materiais manipuláveis.

\begin{abstract}
The present work addresses the main results of a research, whose focus was to present concepts of Spherical Geometry through manipulable materials and activities oriented to undergraduate students in Mathematics. To give theoretical and conceptual support to the research, we considered parameters of the use of manipulable materials in the teaching of mathematics and the teaching of geometry in Brazil. The methodology used sought to reconcile ideas on the use of manipulable materials for the organization of oriented activities that allowed students to make discoveries for themselves. The activities were applied to students of the Mathematics Degree course of a Federal Institute. The research results were qualitatively analyzed using Van Hiele model parameters to describe the performance of those involved. The contribution of the results of this research is inserted in the context of geometry teaching, specifically in the use of manipulable materials in the teaching-learning process.
\end{abstract}

Keywords: Teacher training; Non euclidean geometries; Spherical geometry; Manipulable materials.

\section{Resumen}

El presente trabajo aborda los principales resultados de una investigación, cuyo foco fue presentar conceptos de Geometría Esférica a través de materiales manipulables y actividades orientadas a los estudiantes de la carrera de Grado en Matemáticas. Para dar soporte teórico y conceptual a la investigación, se consideraron parámetros del uso de materiales manipulables en la enseñanza de las Matemáticas y en la enseñanza de la Geometría en Brasil. La 
metodología utilizada buscó conciliar ideas sobre el uso de materiales manipulables para la organización de actividades orientadas que permitieran a los estudiantes realizar descubrimientos por sí mismos. Las actividades fueron aplicadas a estudiantes de la carrera de Licenciatura en Matemáticas de un Instituto Federal. Los resultados de la investigación se analizaron cualitativamente, utilizando parámetros del modelo de Van Hiele para describir el desempeño de los involucrados. El aporte de los resultados de esta investigación se inserta en el contexto de la enseñanza de la Geometría, específicamente en el uso de materiales manipulables en el proceso de enseñanza y aprendizaje.

Palabras clave: Formación docente; Geometrías no euclidianas; Geometría esférica; Materiales manipulados.

\section{Introdução}

Dentre os campos matemáticos existentes, um dos mais importantes desde a antiguidade é a Geometria, que segundo Brasil (1997), é a área da Matemática que investiga o espaço e as formas que podem ocupá-lo. Deste modo, a Geometria encontrase presente em todo o planeta, em suas formas diversas, do micro ao macro.

Entretanto, o ensino de Geometria vem sendo cada vez menos contemplado na educação básica, e este fato deve-se, principalmente, ao despreparo dos professores e à formação deficiente em conteúdo e metodologia para desenvolver satisfatoriamente os processos de ensino e de aprendizagem de Geometria (Carvalho \& Carvalho, 2011).

Nesse contexto situam-se também as Geometrias Não Euclidianas, que se constituem em um marco histórico e importante no desenvolvimento da Matemática Moderna, porém são pouco conhecidas pelos professores, devido ao fato das Geometrias Não Euclidianas geralmente não serem contempladas nos projetos pedagógicos dos cursos de licenciatura em Matemática (Ribeiro, 2012).

Isso impede, muitas vezes, que o futuro professor de Matemática tenha contato com Geometrias diferentes da Geometria Euclidiana Plana e Espacial, principalmente com as Geometrias desenvolvidas no espaço curvo, como a Esférica e a Hiperbólica.

A curiosidade para compreensão do mundo sempre levou a humanidade à busca de novos conhecimentos e à constatação de que esses conhecimentos sempre se renovam. No que se refere ao conhecimento sobre o mundo não euclidiano, sua importância radica em que ele permite a ampliação da visão geométrica, para todos os tipos de Geometria. Deste modo, “[...] o professor de Matemática que tem conhecimento das Geometrias Não Euclidianas terá mais ferramentas para ensinar a euclidiana" (Ribeiro, 2012, p. 32).

Diversas pesquisas brasileiras, dentre as quais pode-se citar Reis (2006), Marqueze (2006), Antunes (2009), Kaleff (2010), Carvalho \& Carvalho (2011), Ribeiro (2012), Leivas (2012), Brum, Schuhmacher \& Silva (2015), apontam um crescimento no interesse da introdução desse tema nos cursos de formação de professores.

Nesse sentido, por se tratar de um ramo importante da Matemática, do ponto de vista histórico e educacional, considerase relevante que as Geometrias Não Euclidianas sejam contempladas nos currículos dos cursos de formação de professores visando contribuir com o desenvolvimento de conhecimentos teóricos, metodológicos, práticos e investigativos dos professores de Matemática, para que possam implementar, satisfatoriamente, os processos de ensino e de aprendizagem dessas Geometrias no contexto da educação básica.

Este trabalho contempla a análise de uma proposta orientada ao ensino de conceitos de Geometria Esférica, centrada na utilização de materiais manipuláveis, desenvolvida no Instituto Federal de Educação, Ciência e Tecnologia do Norte de Minas Gerais (IFNMG), Campus Januária, com os acadêmicos do curso de Licenciatura em Matemática. Essa proposta tem como objetivo verificar a viabilidade do uso de materiais manipuláveis nos processos de ensino e de aprendizagem de conceitos de Geometria Esférica. 


\section{Estudos Preliminares e Metodologias para o Ensino de Geometria Esférica}

Esta seção contempla uma síntese de estudos preliminares relacionados à pesquisa desenvolvida, bem como algumas metodologias que podem ser utilizadas nos processos de ensino e de aprendizagem de Geometria Esférica, as quais foram contempladas na elaboração das atividades e implementação da proposta analisada nesta pesquisa.

Destaca-se primeiramente o uso de materiais manipuláveis que permitem o desenvolvimento da visualização de conceitos geométricos, de forma a evidenciar a aplicabilidade ao ensino de Geometria Esférica. Segundo Reis (2006), a utilização destes materiais permite a manipulação dos modelos geométricos e facilitam a compreensão dos conceitos matemáticos abordados.

Considera-se, neste trabalho, a definição de materiais manipuláveis no sentido proposto por Sousa \& Oliveira (2010, p. 2):

De fato, materiais manipuláveis são objetos, desenvolvidos e/ou criados para trabalhar com conceitos matemáticos de forma que venham a facilitar a compreensão e o desenvolvimento do aluno, de modo que os estudos possam ser realizados de maneira prazerosa.

A utilização de materiais manipuláveis nos processos de ensino e aprendizagem de Geometria é defendido por Leivas (2012, p. 11) ao afirmar que "[...] o uso de materiais concretos, com os quais o estudante possa manusear, realizar transformações e obter conclusões, é de importância capital para desenvolver hábitos de pensamento geométrico". Sobre o uso de planificações e objetos comuns, o autor afirma que esses materiais podem auxiliar no desenvolvimento da Geometria Espacial e possibilitam a introdução de alguns aspectos de Geometrias Não Euclidianas, tornando a Geometria agradável, significativa e atrativa uma vez que o indivíduo a relaciona com o espaço em que vive e com as experiências realizadas.

Marqueze (2006), conclui que o uso de materiais concretos nas atividades em sala de aula, tais como esferas de isopor, barbantes, elásticos, alfinetes, bexigas, réguas flexíveis, compasso, canetas coloridas e outros materiais mais comuns, como lápis e papel, despertam no aluno o interesse e contribuem com a compreensão da Geometria Plana e de outras Geometrias, como a Geometria Esférica, foco desta pesquisa.

Além desses recursos manipuláveis para o ensino de Geometria, ressalta-se a importância do Modelo de Van Hiele para a proposição/elaboração de atividades e análise dos níveis de compreensão dos conteúdos de Geometria por parte dos alunos. Este modelo originou-se nos trabalhos de tese de doutorado do casal holandês Dina van Hiele-Gedolf e Pierre van Hiele, na Universidade de Utrecht, em 1957.

Sobre este modelo, Rodrigues (2015, p. 1) afirma que: “[...] tem sido utilizado para facilitar a compreensão de conteúdos de Geometria enriquecendo o espaço de ensino e aprendizagem". Este modelo consiste em cinco níveis de compreensão, os quais são enunciados e descritos por Crowley (1994) e Rodrigues (2015) da seguinte forma:

- Nível 0: Visualização - consiste na percepção do espaço pelos participantes simplesmente como algo que existe em torno deles, avaliando as figuras geométricas apenas pela aparência.

- $\quad$ Nível 1: Análise - Inicia-se uma análise dos conceitos geométricos, a partir da percepção de características e a descrição de algumas propriedades.

- $\quad$ Nível 2: Dedução Informal - Os participantes conseguem estabelecer inter-relações de propriedades tanto dentro de figuras, quanto entre figuras, e ainda ordenar de forma lógica as propriedades.

- Nível 3: Dedução - Compreende-se a importância da dedução e da teoria geométrica no contexto axiomático. Ainda são percebidos o papel e a inter-relação de termos não definidos, axiomas, postulados, definições, teoremas e das demonstrações. 
- Nível 4: Rigor - O aluno é capaz de trabalhar em vários sistemas axiomáticos, isto é, podem-se estudar Geometrias Não Euclidianas e comparar sistemas.

Além disso, Cardoso (2015) enuncia as propriedades que caracterizam o modelo de Van Hiele da seguinte forma:

- Sequencial: O modelo é parte de uma teoria construtivista, na qual o sucesso em um determinado nível depende do conhecimento adquirido no nível anterior.

- Avanço: O progresso ou o não-progresso de um aluno nesses níveis depende apenas dos conteúdos e métodos de instrução recebidos.

- Intrínseco e extrínseco: Os objetos ligados a um nível se tornam objetos de ensino do próximo.

- Linguística: Cada nível possui símbolos próprios e a estes símbolos está conectado um sistema próprio de relações.

- Combinação inadequada: Para que o aprendizado e o progresso desejados sejam alcançados, o aluno e o curso devem estar no mesmo nível, simultaneamente.

Desse modo, "[...] o método, a organização do curso, do conteúdo e o material utilizado são extremamente importantes" (Cardoso, 2015, p. 6). O modelo ainda propõe cinco fases sequenciais de aprendizagem, segundo Rodrigues (2015): Interrogação/informação, orientação dirigida, explicação, orientação livre, integração. O modelo de Van Hiele não especifica os conteúdos ou currículos a serem trabalhados, podendo ser aplicado à maioria dos conteúdos de Geometria (Cardoso, 2015).

Entende-se que o Modelo de Van Hiele tem potencialidade para o desenvolvimento dos processos de ensino e de aprendizagem de Geometria, especialmente por possibilitar a organização das atividades e a progressão dos participantes através de uma sequência de níveis de compreensão de conceitos (Rodrigues, 2015), e permite a utilização de materiais manipuláveis até o nível 2.

Dessa forma, as atividades que foram propostas e desenvolvidas nesta pesquisa primaram pela utilização de materiais manipuláveis e de instruções que levaram em consideração os níveis 0,1 e 2 do modelo de Van Hiele.

\section{Minicurso: Atividades Propostas e Desenvolvidas}

Esta pesquisa teve como objetivo analisar a viabilidade da implementação de uma proposta didática, baseada na utilização de materiais manipuláveis, para os processos de ensino e de aprendizagem de alguns conceitos de Geometria Esférica. A proposta culminou na elaboração de um minicurso intitulado Geometria Esférica por meio de materiais manipuláveis, que foi desenvolvido posteriormente no Laboratório de Matemática do IFNMG - Campus Januária.

As atividades orientadas foram elaboradas na perspectiva do pensamento geométrico, baseadas na utilização de matérias manipuláveis, na resolução de problemas e levando em conta os processos de comunicação e visualização e o modelo de Van Hiele. Seu desenvolvimento possibilitou que os participantes utilizassem os matérias manipuláveis para auxiliar na visualização dos conceitos, nas respostas às questões propostas e nas discussões ocorridas.

Os materiais manipuláveis utilizados durante a implementação do minicurso foram entregues aos participantes no segundo encontro e utilizados até o último. Cada participante recebeu um kit composto por: uma esfera de isopor, uma régua flexível, um transferidor e quatro canudos. Os outros materiais (barbantes, alfinetes, pincéis, tesouras) foram usados em grupo. Quando foram concluídas as atividades, cada participante pôde levar o kit inicialmente disponibilizado.

Neste artigo, optou-se pela discussão de resultados e análise dos dados de três atividades do minicurso, as quais podem ser apreciadas a seguir. 
A Atividade 1 - Construção de circunferência na Esfera - é composta por cinco etapas, conforme ilustrado na Figura 1.

Figura 1: Construção de circunferências na esfera.

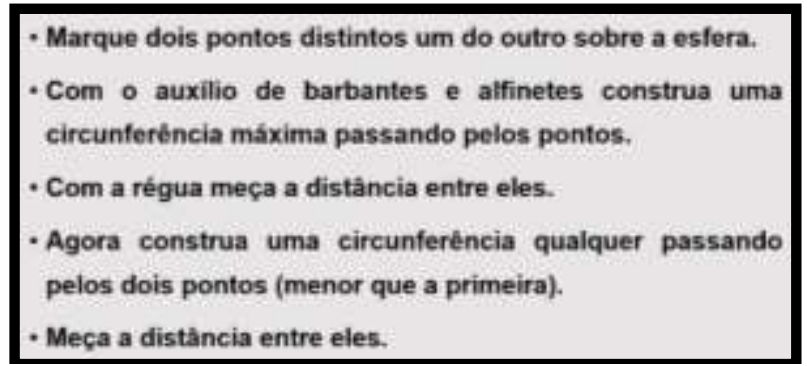

Fonte: Dados da pesquisa.

A Figura 1 detalha o processo de construção de circunferência na esfera, que não é tão óbvio como no plano. Baseado na atividade proposta na Figura 1, os acadêmicos tiveram a oportunidade de discutir os seguintes preceitos teóricos:

Teorema 1: A interseção de uma superfície esférica com um plano passando pelo seu centro é uma circunferência de mesmo centro e raio, como mostra a Figura 2.

Figura 2: Plano interceptando a superfície esférica.

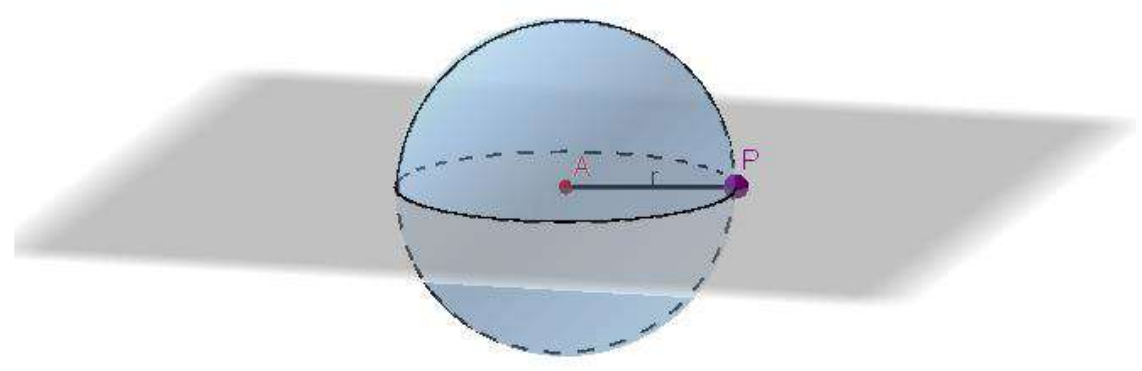

Fonte: Dados da pesquisa

É possível observar na Figura 2 uma circunferência máxima, cuja definição é dada a seguir.

Definição 1: A circunferência do teorema 1 é chamada circunferência máxima, ilustrada na Figura 3 a seguir.

Figura 3: Circunferências máximas na superfície esférica.

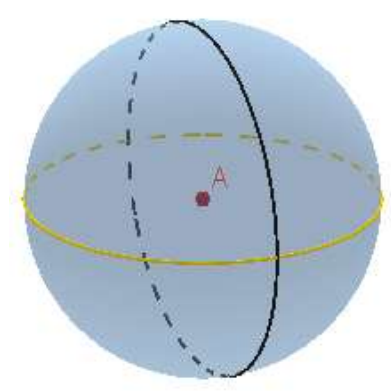

Fonte: Dados da pesquisa 
Na Figura 3 pode-se ver duas circunferências máximas.

Propriedade 1: A menor distância entre dois pontos distintos na esfera é a menor porção da circunferência máxima que os contém (veja o arco de circunferência destacado em amarelo na Figura 4 a seguir).

Figura 4: Menor distância entre os pontos T e U na Circunferência.

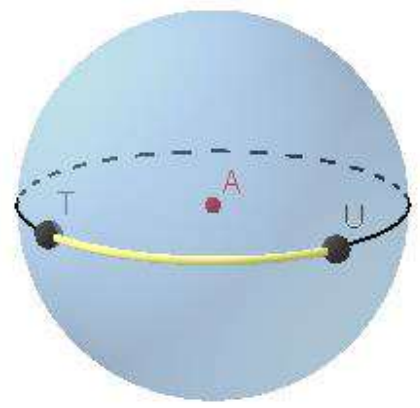

Fonte: Dados da pesquisa

Na Figura 4, observa-se em amarelo um arco da circunferência máxima, que é a menor distância entre dois pontos distintos na esfera.

A Atividade 2 - Soma dos ângulos internos de um triângulo esférico - , constituída por 4 etapas, está descrita na Figura 5.

Figura 5: Construção de retas na esfera.

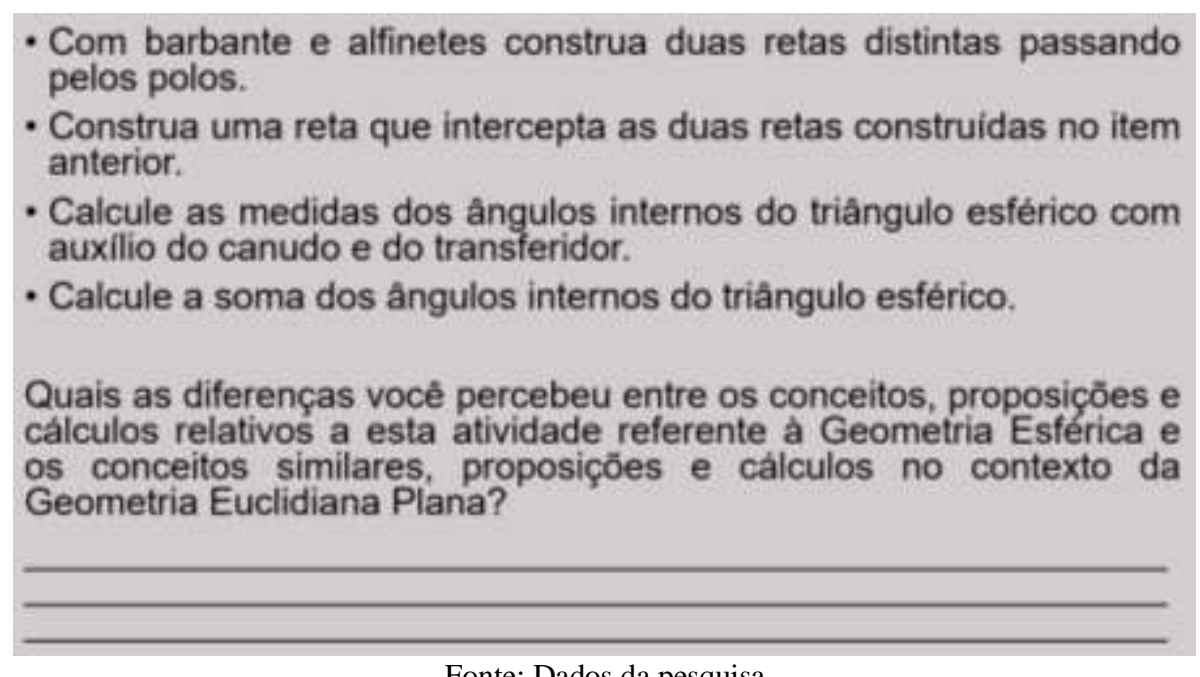

Fonte: Dados da pesquisa

A Figura 5 apresenta as instruções para a construção de retas na esfera. Esta atividade teve como objetivo discutir a propriedade da soma dos ângulos internos do triângulo esférico e obter uma análise e justificativa, por escrito, de cada participante. A Figura 6 apresenta um triângulo esférico. 
Figura 6: Triângulo esférico.

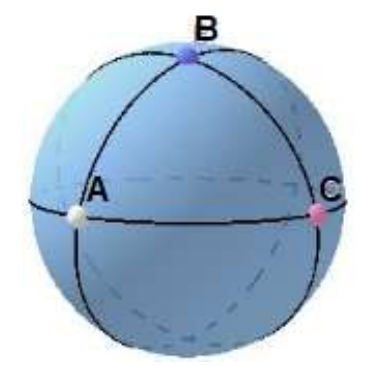

Fonte: Dados da pesquisa

Convém ressaltar que os triângulos esféricos são figuras construídas na superfície esférica (Figura 6) e possuem características próprias.

Segundo Antunes (2009, p. 36), "Dados três pontos, A, B e C, distintos e não pertencentes a uma mesma circunferência máxima, a figura formada pelos arcos de circunferências máximas, que unem esses pontos dois a dois, chama-se triângulo esférico". No trabalho de Zanella (2013) está demostrado o seguinte teorema:

Teorema 2: Se $\alpha, \beta$ e $\gamma$ são as medidas (em radianos) dos ângulos internos de um triângulo esférico, então $\alpha+\beta+\gamma=\pi+$ $\frac{a}{r^{2}}$, na qual $a$ é a área do triângulo e $r$ o raio da superfície esférica.

Esta equação possibilita afirmar que a soma dos ângulos internos de um triângulo esférico é sempre maior do que cento e oitenta graus $\left(180^{\circ}\right)$, pois $\frac{a}{r^{2}}>0$, e é conhecida como a equação de Girard, geômetra francês que a demonstrou essa proposição no ano de 1629 (Zanella, 2013). Esse resultado diverge do que é estudado em Geometria Euclidiana Plana, o que pode gerar surpresa nos alunos após constatarem que nem sempre a soma dos ângulos internos de um triângulo é igual a $180^{\circ}$.

A Atividade 3 - Triângulo Retângulo Esférico - também possuía quatro etapas (Figura 7) e teve por finalidade a aplicação dos conhecimentos até então adquiridos, mas principalmente a aplicação do Teorema de Pitágoras para a validação deste teorema na superfície esférica através da análise dos valores encontrados.

Figura 7: Construção do triângulo na esfera.

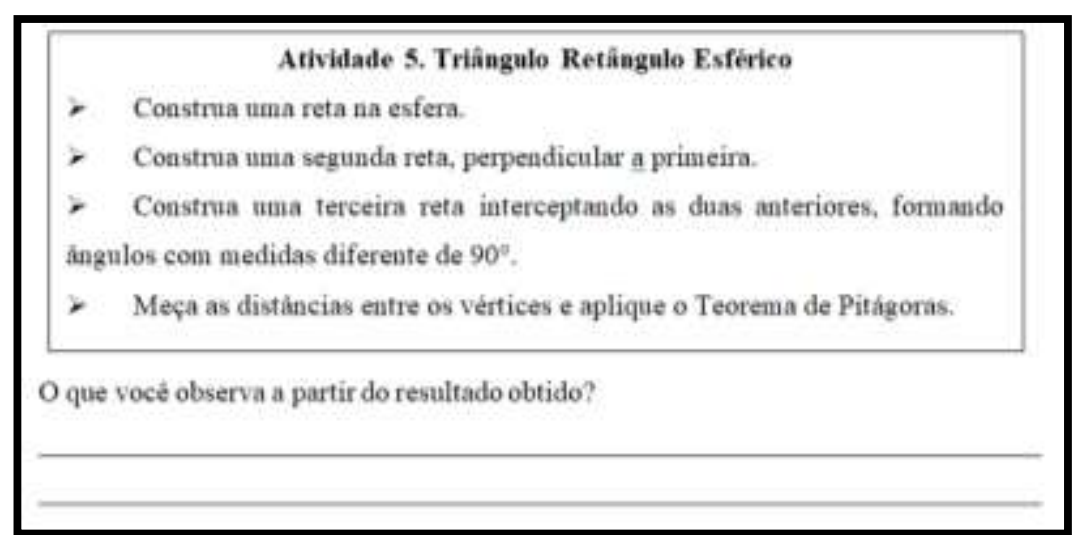

Fonte: Dados da pesquisa.

Na Figura 7, pode-se ver as instruções para a construção de um triângulo retângulo esférico. 


\section{Caminhos Metodológicos}

Para a obtenção dos dados, utilizou-se a observação sistemática das atividades e a aplicação de um questionário, fundamentados em Gil (2008). Na análise dos dados coletados no questionário e nas atividades, a análise qualitativa, caracterizada como análise de conteúdo, que segundo Bardin (2011, p. 44) “[...] é um conjunto de técnicas de análise das comunicações que utiliza procedimentos sistemáticos e objetivos de descrição do conteúdo das mensagens". Embora tenha-se escolhido este tipo de abordagem, foram construídos gráficos e tabelas a partir da quantificação de alguns resultados, o que permitiu aprofundar as análises.

Ressalta-se que esta abordagem qualitativa prevalece em detrimento das quantificações realizadas, pois conforme Gerhardt \& Silveira (2009, p. 32), “A pesquisa qualitativa preocupa-se, portanto, com aspectos da realidade que não podem ser quantificados, centrando-se na compreensão e explicação da dinâmica das relações sociais". Dessa forma, deu-se significado maior as manifestações dos participantes do que aos números obtidos, embora tenha-se usado elementos quantitativos.

As atividades e o questionário foram aplicados no IFNMG - Campus Januária, contando com alunos matriculados no curso de Licenciatura em Matemática que mostraram interesse pelo tema. O Minicurso foi realizado na primeira etapa da pesquisa e, ao final, foram aplicados os questionários destinados a obter uma avaliação dos participantes sobre as atividades e os materiais utilizados durante o desenvolvimento do minicurso. Ressalta-se que no Projeto Pedagógico do curso de Licenciatura em Matemática da referida instituição a disciplina de Geometria Esférica, ou similares, não está incluída, portanto, para os participantes o minicurso constituía-se como novidade. A discussão de resultados relativos às atividades analisadas no minicurso e às questões contempladas no questionário são apresentados nas seções seguintes.

\section{Análise dos Resultados do Minicurso}

Ao término da primeira atividade do Minicurso, que contou com a participação de quinze acadêmicos, observou-se que todos compreenderam, através da visualização e da utilização dos materiais manipuláveis, o fato de que na superfície esférica, a menor distância entre dois pontos é o menor arco de circunferência máxima que passa por eles. O Gráficol apresenta o desenvolvimento dos participantes.

Gráfico 1: Desenvolvimento na Atividade 1.

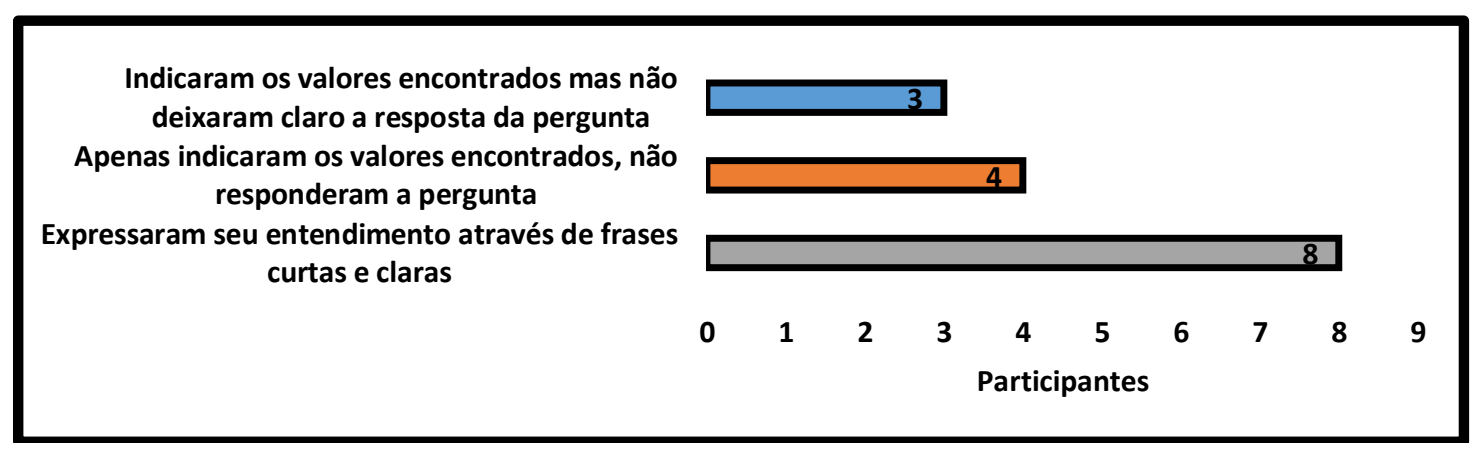

Fonte: Dados da pesquisa

Em relação à atividade 1, cujos dados estão no Gráfico 1, o Aluno A afirmou o seguinte: "Percebemos que a distância entre dois pontos é menor quando passamos pela circunferência máxima do que quando escolhemos uma circunferência menor". E, o Aluno B, declarou que: “A circunferência que formou a menor distância entre os dois pontos foi a maior”. Esse resultado reforça a importância da visualização de conceitos geométricos a partir da manipulação de materiais concretos, corroborando o disposto nos Parâmetros Curriculares Nacionais do Ensino Fundamental (Brasil, 1997) e do Ensino Médio (Brasil, 2011). 
Durante o procedimento de execução da atividade ficou evidente a facilidade dos alunos em interpretar os resultados a partir da interação com os materiais manipuláveis.

Em relação à segunda atividade, que contou com a participação de dezessete acadêmicos, percebe-se que o objetivo foi alcançado, visto que os participantes construíram e verificaram a veracidade da definição sobre o ângulo entre duas retas e o teorema sobre a soma dos ângulos internos de um triângulo esférico ser maior do que $180^{\circ}$, atingindo o segundo nível do modelo de Van Hiele (visualização, análise e dedução informal), conforme descrito por Crowley (1994) e Rodrigues (2015).

O Gráfico 2 contempla uma síntese das respostas dos dezessete participantes referentes à atividade 2.

Dentre as respostas dos participantes relativas às diferenças percebidas entre os conceitos, proposições e cálculos relativos à atividade 2, referente à Geometria Esférica, e os conceitos, proposições e cálculos no contexto da Geometria Euclidiana Plana, destaca-se duas afirmações: De acordo com o Aluno C, "a diferença mais evidente é que a soma dos ângulos internos do triângulo é $223^{\circ}$ e pode variar, diferente da Geometria Plana que é sempre $180^{\circ}$ ”; enquanto que o Aluno D afirmou que "a soma dos ângulos internos de um triângulo, em uma superfície esférica, contradiz um dos teoremas apresentados na Geometria Plana, que afirma, mediante a demonstração, que a soma dos ângulos internos de um triângulo equivale a $180^{\circ}$ ".

Gráfico 2: Desempenho dos alunos no desenvolvimento da Atividade 2.

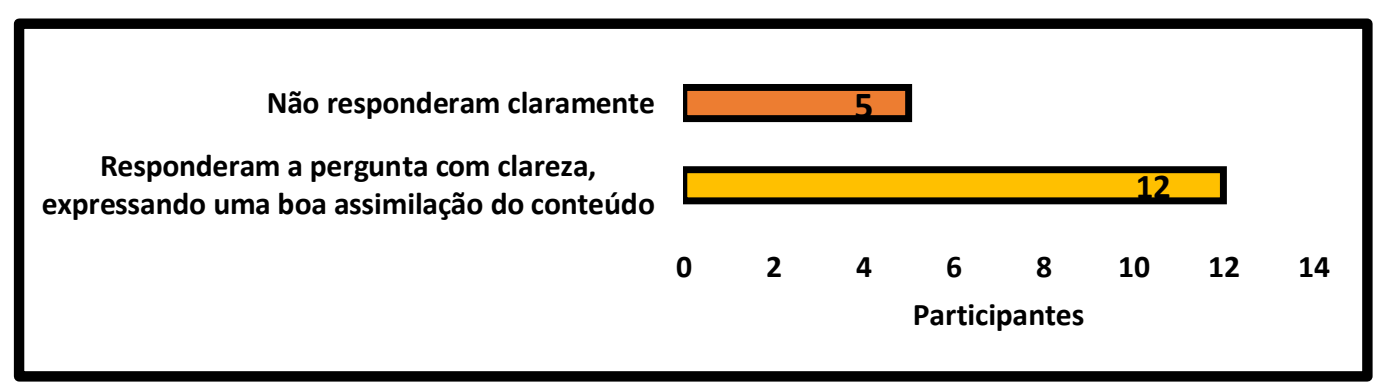

Fonte: Dados da pesquisa

Essas respostas evidenciam que os participantes da pesquisa compreenderam novas proposições relacionados à Geometria Esférica, a partir do desenvolvimento das atividades realizadas no minicurso, com base na utilização dos materiais concretos, desenvolvendo o pensamento geométrico que é importante para o aprendizado, conforme aponta Leivas (2012).

O Gráfico 3 expressa a síntese do desempenho dos participantes na terceira atividade aplicada durante o minicurso, com a presença de dezessete acadêmicos.

Gráfico 3: Desenvolvimento na Atividade 3.

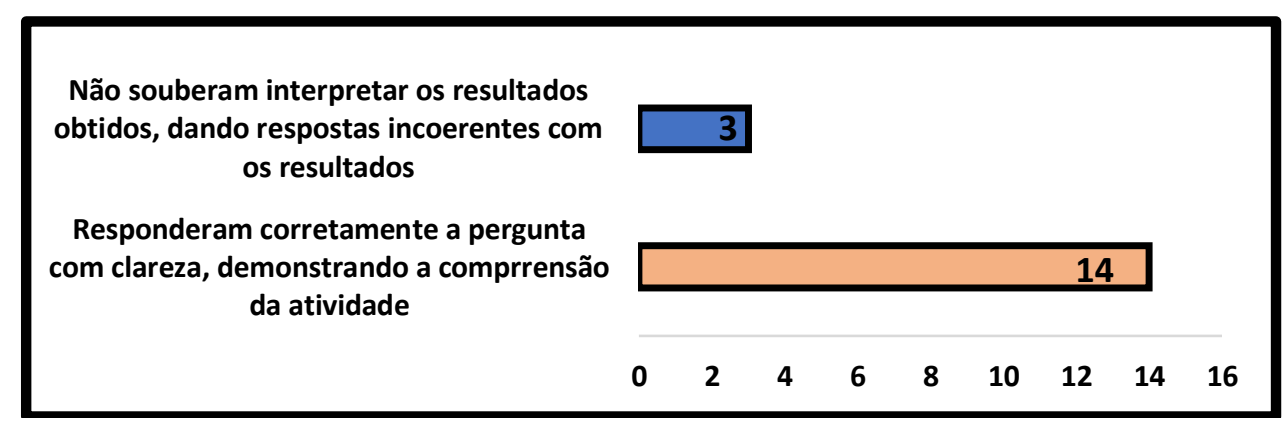

Fonte: Dados da pesquisa.

Embora alguns participantes não tenham compreendido o que a atividade solicitava, pode-se considerar, em virtude do número de acertos e respostas obtidas, que o desenvolvimento foi satisfatório. Alguns relatos dos participantes ilustram essa conclusão, como pode ser contemplado nas respostas de dois participantes que observaram mais detalhadamente a atividade. O 
Aluno D considerou que "o teorema falhou, pois as retas são curvas, logo a soma dos ângulos interno é superior a $180^{\circ}$, e o Teorema de Pitágoras só é válido para triângulos com a soma dos ângulos igual a 180"”; E, o Aluno E, afirmou que: "o Teorema de Pitágoras, quando aplicado em uma superfície esférica, os valores dos termos que se obtém, contradizem a veracidade da igualdade".

Observa-se que alguns participantes relacionaram a curvatura da superfície e a falha do Teorema de Pitágoras, e outros com a soma dos ângulos internos, fato estes que mostram a aquisição do conceito de superfície esférica e da soma dos ângulos internos de um triângulo esférico através das atividades práticas. Novamente percebe-se o desenvolvimento do pensamento geométrico dos alunos, que alcançaram o segundo nível do modelo de Van Hiele (Crowley, 1994; Rodrigues, 2015).

\section{Análise do Questionário}

Ao final do minicurso foi aplicado um questionário com a finalidade de avaliar a metodologia utilizada. A discussão dos resultados relativos às questões do questionário está descrita a seguir.

A primeira questão apresentava o seguinte enunciado: As atividades desenvolvidas durante o minicurso lhe proporcionaram o conhecimento e a compreensão das diferenças entre a Geometria Euclidiana e as Geometrias Não Euclidianas?

Todos os participantes que responderam ao questionário compreenderam as diferenças entre as Geometrias após realizarem as atividades propostas. Segundo o Aluno A, "as comparações feitas entre esses dois tipos de Geometria, foram essenciais, pois me auxiliaram a compreender melhor as suas diferenças".

O termo curiosidade apareceu em várias falas dos participantes, conforme as descrições seguintes:

- Foi possível conhecer as diferenças, despertando curiosidades, tirando as dúvidas e contribui para um aprendizado melhor (Aluno A).

- Em toda a minha trajetória acadêmica, sempre estudei apenas conceitos de Geometria Euclidiana, então durante o minicurso foi possível compreender um pouco dessas Geometrias Não Euclidianas. É claro que não esgotamos o assunto, mas aumentou nossa curiosidade para estudar melhor o assunto (Aluno B).

Este fato possibilita inferir que a curiosidade pode mobilizar os conhecimentos do aluno e, consequentemente, contribuir com a aprendizagem dos objetos matemáticos estudados. Nesse sentido, corrobora-se a afirmação de Marqueze (2006), ao considerar que o conhecimento adquirido por meio do uso de materiais manipuláveis instiga o indivíduo a ser curioso, a buscar compreender o conceito construído materialmente e desperta o interesse do aluno para a compreensão de novos objetos matemáticos.

A próxima pergunta tinha como objetivo observar se as atividades desenvolvidas ao longo do minicurso proporcionaram o aprendizado dos conceitos de Geometria Esférica. Todos os participantes responderam que sim. As falas dos participantes justificam esse resultado, algumas das quais foram sintetizadas no Quadro 1. 
Quadro 1: Narrativas dos participantes sobre as atividades desenvolvidas.

\begin{tabular}{|l|l|}
\hline $\begin{array}{l}\text { "Elas proporcionaram a verificação da veracidade } \\
\text { das informações que estavam sendo explicadas". } \\
\text { (Aluno A) }\end{array}$ & $\begin{array}{l}\text { "Porque elas nos permitiram compreender com mais } \\
\text { clareza alguns conceitos e identificar algumas } \\
\text { diferenças da Geometria Euclidiana e das Não } \\
\text { Euclidianas". (Aluno C) }\end{array}$ \\
\hline $\begin{array}{l}\text { "Compreendi muitas coisas que não consegui } \\
\text { quando estudei esfera em Geometria. O fato de } \\
\text { trabalharmos com os materiais manipuláveis e e } \\
\text { fazendo as atividades na prática ajudou na } \\
\text { compreensão". (Aluno D) }\end{array}$ & $\begin{array}{l}\text { "Tivemos uma ótima noção do que é a Geometria e em que utilizá-la. Também achei } \\
\text { interessante a visão sobre a esfera do globo terrestre } \\
\text { na Geometria Esférica". (Aluno E) }\end{array}$ \\
\hline
\end{tabular}

Fonte: Dados da pesquisa

Portanto, as atividades desenvolvidas alcançaram o objetivo pretendido, reforçando a importância da contextualização e da prática na compreensão dos conceitos matemáticos, como pode-se observar nas respostas do Quadro 1.

Além de avaliar as atividades aplicadas, foi solicitado aos participantes que analisassem os recursos utilizados no decorrer do minicurso que se restringiram aos materiais manipuláveis, figuras e slides. A análise desses recursos revela-se importante na avaliação da qualidade do minicurso.

A avaliação foi feita mediante a atribuição de notas, pelos participantes, a estes materiais, numa escala de zero a dez, em que zero indica satisfação mínima e dez indica satisfação máxima, acerca do auxílio desses recursos na compreensão dos conceitos de Geometria Esférica. No Gráfico 4 está expressa a avaliação dos materiais manipuláveis utilizados, realizada por dezoito participantes que estavam presentes no último encontro do minicurso.

A nota sete foi atribuída por um participante que explicou o motivo da sua avaliação: "O material manipulável foi muito bom, porém muitas vezes os barbantes saíam do lugar, a esfera não era totalmente regular e isso dificultou um pouco a minha aprendizagem".

Gráfico 4: Avaliação dos materiais manipuláveis.

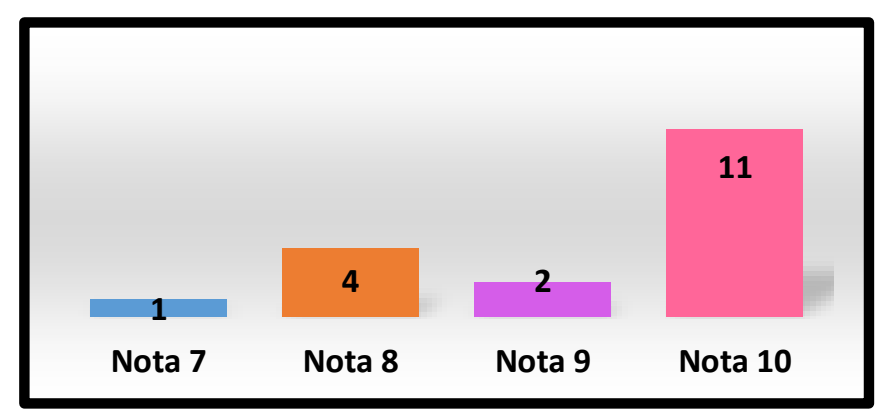

Fonte: Dados da pesquisa.

O fato de as esferas não serem regulares só foi descoberto durante a realização das atividades, na qual observou-se o defeito de fabricação. Infelizmente não foi possível realizar a substituição desse material durante o minicurso. Os barbantes saíam do lugar devido à falta de atrito com a esfera de isopor. Dessa forma, para contornar o problema, alguns participantes construíam as retas com o auxílio da régua flexível e de pincéis ao invés de barbantes e alfinetes, conforme mostra a Figura 8. 
Figura 8: Construção de retas com pincel e régua flexível.

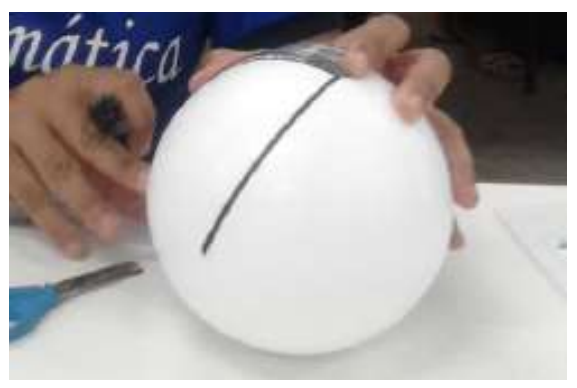

Fonte: Dados da pesquisa

A solução encontrada para substituir os barbantes, como pode-se ver na Figura 8, foi uma boa iniciativa. A média ponderada das notas atribuídas aos materiais foi igual a 9,3. Este valor mostra que os materiais avaliados receberam uma ótima nota, o que significa que foram eficazes para auxiliar os alunos na compreensão dos conceitos de Geometria Esférica.

Com relação aos materiais utilizados no minicurso, os participantes foram indagados sobre a possibilidade de utilizálos em suas futuras aulas de Matemática, pois um aspecto importante da aquisição de novos conhecimentos é o de não o deixar estagnado. Considera-se importante que o futuro professor de Matemática continue estudando o tema e aplicando seus conhecimentos em suas futuras atividades profissionais.

Observa-se que todos os participantes que responderam à pergunta marcaram a opção Sim, e em suas justificativas ressaltaram grande interesse em levar as atividades desenvolvidas para a sala de aula. Alguns alunos relataram que:

- Sim, pois é um conhecimento novo, interessante e deve ser passado adiante (Aluno A).

- Por que são conhecimentos que não podem deixar de ser utilizados, pois enriquece tanto os alunos quanto os educadores (Aluno B).

Nota-se que os participantes citam a importância da contribuição do novo conhecimento para a formação dos alunos da Licenciatura em Matemática, de modo que "Todo conhecimento deve ser transmitido enriquecendo as aulas, formando cidadãos capazes de discernir" (Aluno C).

Quanto ao material utilizado, o Aluno D escreveu: "Pois é um material que deve ser compartilhado para os outros com a finalidade de mostrar os diferentes tipos de Geometria e suas aplicações”. Dessa forma, conclui-se que os materiais utilizados no minicurso são didáticos e que os participantes consideram que é possível sua inserção na sala de aula de Matemática no contexto da educação básica.

Quando questionados sobre sugestões/críticas e opiniões sobre o trabalho, os participantes se mostraram interessados no aprofundamento do tema, almejando outros minicursos com carga horária maior com o intuito de alcançarem mais qualificação para lecionarem. Segundo o Aluno E, a inserção de conceitos de Geometrias Não Euclidianas nos cursos de formação de professores de Matemática “[...] contribuirá para a formação de profissionais mais qualificados”. Essa afirmação corrobora a ideia de Ribeiro (2012), no sentido de que conhecendo as Geometrias Não Euclidianas, o professor de Matemática terá mais ferramentas para ensinar outras Geometrias.

\section{Considerações Finais}

O minicurso proporcionou aos licenciandos o conhecimento das Geometrias Não Euclidianas, sua origem e desenvolvimento, conceitos, propriedades e aplicações, e uma metodologia de inserção desse tópico, em sala de aula, por meio da utilização de materiais manipuláveis. Portanto, considera-se que o objetivo do estudo foi alcançado. 
Com relação ao objetivo de verificar a viabilidade do uso de materiais manipuláveis na consolidação de conceitos de Geometria Esférica, nota-se, por meio dos dados apresentados, relativos às atividades realizadas e às falas dos participantes, que os materiais utilizados auxiliaram no aprendizado dos conceitos contemplados nas atividades, sendo atribuídas notas satisfatórias pelos futuros professores de Matemática (média ponderada de 9,3 pontos em um total de 10 pontos). Esse resultado evidencia a potencialidade da utilização de materiais manipuláveis para o desenvolvimento de atividades referentes aos processos de ensino e de aprendizagem de Geometrias Não Euclidianas no contexto da formação de professores de Matemática, uma vez que a relevância do minicurso foi considerada satisfatória a todos os participantes.

$\mathrm{O}$ trabalho desenvolvido revelou-se potencialmente multiplicador, uma vez que os participantes, alunos do curso de Licenciatura em Matemática, tiveram contato com um novo conteúdo e novas metodologias para trabalhar conceitos e propriedades de Geometria Esférica por meio das atividades práticas desenvolvidas no minicurso. O fato de que todos pretendem ensinar este conteúdo em suas aulas reforça a importância atribuída pelos participantes tanto aos objetos matemáticos quanto à metodologia utilizada no minicurso.

A partir dos resultados obtidos nesta pesquisa, propõe-se a ampliação e o aprofundamento das investigações relativas ao desenvolvimento dos conceitos, proposições, aplicações teóricas e práticas e de metodologias que possam se revelar satisfatórias para o desenvolvimento dos processos de ensino e de aprendizagem das Geometrias Não Euclidianas e de suas conexões/articulações intramatemáticas e extramatemáticas no contexto da Licenciatura em Matemática.

Propõe-se ainda o aprofundamento nas pesquisas relativas à elaboração, implementação e análise de atividades e/ou sequências didáticas centradas tanto em Geometria Euclidiana quanto em Geometrias Não Euclidianas, contemplando todos os cinco níveis propostos no Modelo de Van Hiele, buscando atingir, inclusive, os níveis de dedução e rigor necessários aos desenvolvimento dos conhecimentos didático-matemáticos e de competências necessários ao professor que ensina Matemática nos distintos níveis e modalidades.

\section{Referências}

Antunes, M. C. (2009). Uma possível inserção das geometrias não euclidianas no ensino médio (Monografia de Graduação). Universidade Federal do Rio Grande do Sul, Instituto de Matemática.

Bardin, L. (2011). Análise de conteúdo. Edições 70.

Brasil. (1997). Ministério da Educação. Secretaria de Educação Fundamental. Parâmetros curriculares nacionais: matemática: $1^{\circ}$ e $2^{\circ}$ ciclos do ensino fundamental. MEC/SEF.

Brasil. (2011). Ministério da Educação. Secretaria de Educação Média e Tecnológica. Parâmetros curriculares nacionais: ensino médio. MEC/SEF.

Brum, W. P.; Schuhmacher, E. \& Silva, S. C. R. (2015). As geometrias esférica e hiperbólica em foco: sobre a Apresentação de alguns de seus Conceitos Elementares a Estudantes do Ensino Médio. Bolema: Boletim de Educação Matemática, 29 (51), 419-427.

Cardoso, E. J. (2015). Teoria de Van Hiele aplicada ao ensino de funções. In Anais, 19 Encontro de Estudantes de Pós-Graduação em Educação Matemática (pp. 1-12). Juiz de Fora: Universidade Federal de Juiz de Fora.

Carvalho, M. A. S., \& Carvalho, A. M. F. T. (2011). O ensino de geometria não euclidiana na educação básica. In Anais, 13 Conferência Interamericana de Educação Matemática (pp. 1-10). Universidade Federal de Pernambuco.

Crowley, M. L. (1994). O modelo de Van Hiele de desenvolvimento do pensamento geométrico. In Lindquist, M. M. \& Shulte, A. P. (Orgs.). Aprendendo e ensinando geometria (pp. 1-20). Atual.

Gerhardt, T. E. \& Silveira, D.T. (2009). Métodos de pesquisa. Editora da UFRGS.

Gil, A. C. (2008). Métodos e técnicas de pesquisa social. (6a. ed.). Atlas.

Kaleff, A.M. M. R. (2010). Geometrias não euclidianas na educação básica: utopia ou possibilidade? In Anais, 10 Encontro Nacional de Educação Matemática (pp. 1-17). SBEM.

LEIVAS, J. C. P. (2012). Educação geométrica: reflexões sobre ensino e aprendizagem em geometria. Educação Matemática em Revista - RS, 1 (13), 9-16. 
Research, Society and Development, v. 10, n. 1, e18510111646, 2021 (CC BY 4.0) | ISSN 2525-3409 | DOI: http://dx.doi.org/10.33448/rsd-v10i1.11646

Marqueze, J. P. (2006). As faces dos sólidos platônicos na superfície esférica: uma proposta para o ensino-aprendizagem de noções básicas de geometria esférica (Dissertação de Mestrado). Pontifícia Universidade Católica de São Paulo,

Paulo.

Reis, J. D. S. (2006). Geometria esférica por meio de materiais manipuláveis (Dissertação de Mestrado). Universidade Estadual Paulista, Instituto de Geociências e Ciências Exatas, Programa de Pós-Graduação em Educação Matemática.

Ribeiro, R. D. G. L. (2012). O ensino de geometrias não euclidianas: um olhar sob a perspectiva da divulgação científica (Dissertação de Mestrado). Universidade de São Paulo, Programa de Pós-Graduação em Educação, Área de concentração Ensino de Ciências e Matemática.

Rodrigues, A. C. (2015). O modelo de Van Hiele de desenvolvimento do pensamento geométrico (Trabalho de Conclusão de Curso de Graduação). Universidade Católica de Brasília, Curso de Matemática.

Sousa, G. C.; Oliveira, J. D. S. (2010). O uso de materiais manipuláveis e jogos no ensino de matemática. In Anais, 10 Encontro Nacional de Educação Matemática (pp. 1-11). SBEM.

Zanella, I. A. (2013). Geometria esférica: uma proposta de atividades com aplicações (Dissertação de Mestrado). Universidade Estadual de Londrina, Centro de Ciências Exatas, Programa de Pós-Graduação em Matemática. 